Tatiana VICTOROFF

Université de Strasbourg

\title{
Misteri d'Elx et Mystère de la Dormition de la Mère de Dieu de Dimitri de Rostov : entre représentation et présentation
}

Parmi les nombreux mystères de l'Assomption, répandus pratiquement à travers toute l'Europe médiévale (on peut citer par exemple les textes anglo-normands joués à Bayeux en 1351 ou à Coutances en 1411, les textes français joués à Rodez au XVI $\mathrm{s}$, catalans à Tarragone au $\mathrm{XV}^{\mathrm{e}} \mathrm{s}$, ou, pour ne pas aller plus loin, à Elx à partir du XV $\mathrm{XV}^{\mathrm{e}}$ ou même du XIII ${ }^{\mathrm{e}}$ s.), il nous semble intéressant de nous arrêter sur un exemple russe datant du $\mathrm{XVII}^{\mathrm{e}}$ s. à Rostov, où, en l'absence presque totale d'un théâtre des mystères dans la Russie ancienne, l'apparition d'un Mystère sur ce sujet paraît assez étonnante. Et ce, pour deux raisons : la première étant la réticence très forte de l'orthodoxie, fondée sur des motifs théologiques, à l'égard du théâtre, même chrétien, la seconde parce que ce mystère est l'œuvre d'un clerc de haut rang, le métropolite Dimitri de Rostov, de son vrai nom, Daniel Touptalo (1651-1709), un personnage public et auteur d'écrits spirituels, célèbre pour ses «Vies de Saints » (Tcheti-Minei) qui sont entrées dans le corpus liturgique essentiel de l'Eglise orthodoxe. Ce grand homme se révèle aussi un grand amateur de théâtre, puissant moyen d'enseignement et de formation à ses yeux, raison pour laquelle il a créé le théâtre scolaire de l'Académie théologique de $\operatorname{Rostov}^{1}$ où ont été montés ses mystères.

L'histoire de la découverte du texte du Mystère de la Dormition est un peu moins mystérieuse que celle de celui d'Elx (apparu triomphalement, selon la légende, du côté de la mer), mais reste énigmatique : il a été retrouvé au début du $\mathrm{XX}^{\mathrm{e}} \mathrm{s}$. parmi les manuscrits de la Bibliothèque Synodale de Moscou, trois siècles après sa rédaction. C'est assez tardif et très peu nombreuses sont les recherches autour de ce texte qu'il nous paraît intéressant de mettre en parallèle avec son prédécesseur catalan, que nous prendrons ici comme modèle du mystère occidental de l'Assomption. Et ce d' 'autant plus que Dimitri de Rostov est connu pour être un grand esprit, très ouvert à la culture occidentale, et qu'il connaissait sans nul doute le théâtre des mystères européens, en particulier dans sa version polonaise qui avait pénétré en Ukraine.

L'analyse qui suit, construite comme une comparaison des deux textes, va montrer comment les différents accents dans la perception de la même fête chrétienne engendrent des différences dans la poétique et la scénographie de la représentation théâtrale de l'Assomption dans les deux traditions et ce, dans l'intitulé même des textes.

\section{L'évènement central et son encadrement : "la gloire » ou « la maternité mystique »}

Dans le traitement du même sujet, l'Assomption (ou Dormition de la Mère de Dieu selon la tradition orthodoxe), les deux auteurs s'appuient sur un procédé classique du mystère médiéval, c'est à dire l'entrelacement typologique de divers épisodes de l'histoire sacrée autour de l'événement clé. Cependant, chacun des deux textes fait appel au fond propre de sa tradition spirituelle particulière. L'auteur anonyme du mystère d'Elx se fonde sur l'histoire de l'Assomption telle qu'elle est exposée dans La Légende dorée de Jacques de Voragine. Dimitri de Rostov reprend et adapte son propre texte des «Vies des Saints» (Tcheti-Minei). Les deux auteurs travaillent assez librement avec leurs sources : la structure dramaturgique, le choix des épisodes, des personnages et le dispositif scénique révèlent leurs aspirations particulières.

\footnotetext{
${ }^{1}$ Selon Dimitri de Rostov, les représentations religieuses « développaient chez les étudiants la piété et la sage audace », in Shliapkin I., A. Saint Dimitri de Rostov et son époque (1651-1709), SPb, 1891, c. 347.
} 
La description de l'événement central reste sensiblement la même, mais tout ce qui se déroule à Elx sur deux jours de façon si spectaculaire, est formulé dans le texte de Dimitri de Rostov par un seul personnage dans un monologue au premier acte. En revanche, l'encadrement de cet épisode est ici significatif. La Dormition russe commence bien avant les évènements évangéliques : le premier acte s'ouvre sur la prophétie de Jacob qui voit son rêve déchiffré par un Ange ( Cette échelle sera pour le monde un pont vers les cieux $»^{1}$,acte I, p. 5), ce qui est une préfiguration du rôle de la Mère de Dieu dans le salut de l'homme. Dans la deuxième partie, dans la chaîne d'épisodes qui élargissent l'action, apparaît, à la différence du mystère d'Elx, non pas une foule de juifs qui se convertiront, mais un seul pêcheur, jugé par le tribunal céleste et sauvé par l'intercession de la Mère de Dieu (le mystère se mêle ici à des éléments du genre de la moralité). Ainsi, cet encadrement met en perspective l'histoire de Marie, qui n'est pas une histoire privée et purement «humaine»; elle ne s'inscrit pas uniquement dans la narration évangélique, mais aussi dans les prophéties de l'Ancien Testament, et participe réellement à chaque destin humain. La Mère de Dieu chez Dimitri de Rostov est une mère universelle. Par son action elle peut faire l'impossible, comme obtenir le salut d'un condamné dans les cieux - un thème qui revient dans la littérature russe à travers les siècles chez Tioutchev, Dostoïevski, et plusieurs autres, et dont on trouve le prototype dans le fameux récit apocalyptique russe du XIII ${ }^{\mathrm{e}} \mathrm{s}$. « Le Pèlerinage de la Mère de Dieu parmi les tourments », où elle tombe à genoux, "pleure et ne veut pas se consoler », prie Dieu de faire miséricorde à tous sans distinction, et réussit à adoucir leur châtiment. Pour comprendre la particularité de cette puissance d'intercession, il suffit de citer quelques textes médiévaux occidentaux qui touchent à la même problématique, comme le «Mystère du Jugement Dernier» $\left(\right.$ France, $\left.\mathrm{XIV}^{\mathrm{e}} \mathrm{s}\right)$, où la prière de la Vierge pour les pécheurs est impuissante face à la Justice qui exige leur condamnation.

La maternité mystique, qui dans le mystère d'Elx ne se manifeste que dans le final au moment de la vénération de la Vierge, est donc au centre du mystère de Dimitri de Rostov ; l'ascension, qui demeure invisible dans le mystère russe, n'est que le début de la mission propre de la Mère de Dieu. Sur ce chemin elle est accompagnée, non des deux Maries du Mystère d'Elx (qui dans la tradition occidentale sont ses compagnes fidèles depuis le Dialogue au Sépulcre (IX $\mathrm{s}$.), mais par les personnifications de Pleurs, Consolation et «Vravij » (le «Rameau du Paradis » en slavon), personnages traditionnels de la poésie spirituelle russe. Pleurs se lamente sur la perte de la grâce divine, en la personne de la Vierge, mais trouve sa Consolation dans le fait que Marie sera, dès maintenant, l'avocate des hommes dans le ciel. Le rôle symbolique de $\operatorname{Vravij}^{2}$, comme dans le mystère d'Elx (où il est transmis de l'Ange à la Vierge, puis à Saint Jean), est une invitation à rejoindre le Christ. Mais ici Vravij a une fonction supplémentaire : doué de parole, il cite le discours de la Mère de Dieu, en tant que témoin direct de sa conversation mystérieuse avec son Fils. Il est vrai qu'il est difficile de trouver un témoin plus direct que le Rameau transmis, mais on peut se demander pourquoi il est besoin d'avoir un intermédiaire pour prononcer les paroles de Marie. Il n'est pas anodin que Dimitri de Rostov ait réécrit cet épisode par rapport à ses «Vies des Saints », où la Mère de Dieu parle à la première personne.

\section{« La foi est ce que nous ne voyons pas $»^{3}$}

\footnotetext{
1 "Та лестница к небеси мостом будет миру", in Dimitri de Rostov. Le Drame de la Dormition (publication de M. Speranskij), Moscou, 1907, p. 5 (141-165).

2 "Светоносное вравие, ветвь фиников из Божьего рая принесенная" // Tcheti-Minei de métrop. de Rostov, 1794, 15 août, la Fête de la Dormition.

3 «вера есть то, что мы не видим, веруем же несомненно», in Enquête sur les croyances secrètes des vieux croyants, sur leur enseignement, sur leur actes et témoignages que peur foi est erronée, leur enseignement perfide et leurs actes non agréables à Dieu (Rosysk o raskol'ničej brynskoj vere, o učenii ih, o delah ih i Iz' âvlenie čto vera ih neprava, učenie ih duševredno i dela ih ne bogougodny)
} 
La réponse vient avec l'apparition d'un autre personnage, Thomas, qui montre en même temps comment les buts liturgiques se mêlent dans le mystère de Dimitri de Rostov avec une fonction politique, en réponse à l'actualité, et en s'éloignant de sa propre narration dans les «Vies des Saints $»^{1}$. Thomas, on le sait, «n'est pas comme les autres ». La tradition apocryphe le montre en retard aux funérailles et doutant du miracle de l'Assomption (épisode qui, à la suite de Jacques de Voragine, est entré dans le corpus du mystère d'Elx, mais sans que ses doutes soient reproduits). Dimitri de Rostov, au contraire, insiste sur ce point : son Thomas ne croit pas même à la mort de la Mère de Dieu («car selon lui elle a supprimé l'aiguillon de la mort» ${ }^{2}$. Dimitri de Rostov introduit trois nouveaux personnages chargés de le convaincre : la Foi, l'Espérance et de la Miséricorde (et ici le texte se transforme, en raison de ces allégories, en moralité), mais leurs arguments n'ont aucun succès auprès de Thomas ; la conviction de ce dernier reste inflexible-: « si je vois, je croirai ». En fait, ce n'est qu'après que l'Ange, désespéré de le convaincre par la parole, lui eut montré la Mère de Dieu «auprès de la Trinité en gloire » ${ }^{3}$ que Thomas se repend: «Je vais l'annoncer aux autres / Afin que nul n'hésite dans la foi / et ne se limite pas aux mystères bien visibles $»^{4}$.

L'entêtement de Thomas, encore une fois manifesté, met en évidence, sous la plume de Dimitri de Rostov , une polémique forte avec les vieux croyants, particulièrement nombreux dans la région de Rostov $^{5}$, auxquels l'auteur reproche leur obscurantisme et leur attachement au rite. Sa réécriture de sa propre source n'est ici pas seulement dramaturgique : c'est une façon d'entrer publiquement en lutte contre l'hérésie qui obscurcit l'intelligence de ses contemporains et de rendre son attaque plus directe et plus efficace. Le repentir de Thomas sonne comme un appel aux vieux croyants, qui, d'après lui, ne croient qu'à ce qui « se voit avec les yeux et se touche avec les mains » («очами зрится и руками осязается») ${ }^{6}$.

\section{Le dispositif scénique}

Ce thème a sa réalisation scénique : l'objet de foi, l'invisible n'est pas montré, il est laissé à l'effort intellectuel, qui s'élève de ce qui est entendu à ce qui n'est accessible qu'à la pensée. Ceci explique la nécessité de présenter le discours de la Mère de Dieu à la troisième personne : tout ce qui demeure dans une « autre dimension » (comme la conversation miraculeuse de la Mère de Dieu avec son Fils, ou l'intercession de la Mère de Dieu pour un pécheur au ciel) n'est pas mis en scène. La Mère de Dieu elle-même n'apparaît pas une seule fois comme personnage, et reste invisible et inaudible - mais le spectateur connaît les résultats de ses actions par les autres - les témoins (comme le Rameau) ou l'Ange, le messager divin.

Ainsi, sa présence reste sensible presque tout au long du texte: elle agit à travers les personnages (on voit le résultat de son intersession dans la conversion miraculeuse du pécheur) ; ses paroles sont largement citées par les autres. Quand l'apparition de la Mère de Dieu devient indispensable (comme dans la scène de l'Apothéose de la Mère de Dieu, où tous les personnages lui

\footnotetext{
${ }^{1}$ Selon les Tcheti-Minei de Dimitri de Rostov, l'apôtre Thomas, " par la volonté de Dieu », n'était pas présent à l'enterrement de la Mère de Dieu. (Povestvovanie ob Uspenii Presvâtoj Bož'ej Materi // Tcheti-Minei, 1794).

2 « яко смертное жало в зачатии стерла», op. cit (734)

3 «зрит Деву с Троицей Божества во Славе», ор. cit

4 «Иду убо прочим сие возвещати / Да никто же ся будет в вере колебати / Да не мудрствуют таинств явных токмо бе», Mystère de la Dormition, op. cit, p. 22-23.

5 Rostov se trouvait située à un carrefour de voies commerciales et administratives («За Волгой 77 вер», dit un proverbe). Le métropolite Dimitri voyait dans le schisme le résultat de l'ignorance se faisant passer pour de l'intelligence : «... некая особая изобретается вера, и уже о вере и простые мужики, и бабы догматизируют и учат..» (Shliapkin I., Op. cit. p. 413).

${ }^{6}$ Le Métropolite Dimitri a écrit, contre ce schisme (raskol), un ouvrage massif, Enquête sur les croyances secrètes des vieux croyants, sur leur enseignement, sur leur actes et témoignages que leur foi est erronée, leur enseignement perfide et leurs actes non agréables à Dieu
} 
offrent leurs dons et s'adressent directement à elle), on apporte sur scène son icône ${ }^{1}$, mettant en évidence sa présence grâce à cette "fenêtre vers l'invisible », selon la définition de l'icône que donne Pavel Florenski. La différence est grande avec la montée triomphale, dans le mystère catalan, de la statue de la Vierge sous la coupole qui s'ouvre sur une pluie d'or et l'apparition de la Trinité Divine sous un aspect sensible.

Les intentions des deux auteurs sont ici manifestes : face à la «tentation générale de la chrétienté à la visualisation et à la matérialisation du mystère $»^{2}$, chaque tradition élabore son propre langage. Dans le mystère de Dimitri, il n'y a ni «lumière de la gloire divine", ni "brusque coup de tonnerre", ni Trinité Divine, mais un récit métaphorique fondé sur des correspondances. Si le Mystère d'Elx est «joué par les personnages » (la caractérisation la plus fréquente du mystère médiéval occidental), le Mystère de Dimitri de Rostov est plutôt « dit par les personnages ». Malgré ces différences, l'intention de la scène finale reste très proche : les deux spectacles s'ouvrent vers le public qui est constitué par la société des croyants en l'invitant à vénérer l'icône ou la statue sur la scène, où chacun se trouve donc invité. L'auteur russe comme l'auteur catalan posent un acte de foi, même s'ils utilisent des moyens différents pour s'approcher du mystère chrétien.

\section{Re/présentation et Représentation}

Cette tension entre représentation et présentation de la réalité sacrée qui est au cœur du théâtre spirituel, nous pouvons l'observer à tous les niveaux (poétique et scénographique). Chaque texte exprime une de des tendances qui remontent aux diverses façons d'exposer la vérité spirituelle. Si pour une tradition, montrer la séparation de l'âme et du corps, ou l'apparition du divin dans la gloire, est possible en langage théâtral, pour la seconde, le mystère de la foi et de la divinité demeure "secret, caché des spectateurs", l'essentiel étant transmis par la parole. Au lieu d'une action grandiose qui englobe le public, elle insiste sur la «transfiguration intérieure », illustrée dans le texte par l'histoire d'un pécheur.

Les évènements placés au centre de chaque texte le soulignent clairement : dans le mystère d'Elx, il s'agit du couronnement de la Vierge, où se rejoignent les sens sémantique (la manifestation du triomphe) et scénographique (le croisement des deux mouvements verticaux (la montée d'Araceli, l'autel céleste, et la descente de la Trinité), tandis que Dimitri de Rostov construit son texte autour de la participation de la Mère de Dieu à la vie humaine : le mouvement vertical est aussi essentiel, mais reste toujours intérieur mental.

L'intitulé de chaque fête formule cette divergence de façon éloquente : l'Assomption au ciel est un terme spatial pour exprimer l'élévation la plus haute, le digne achèvement de la vie de la Vierge, tandis que la Dormition dit un état, suggère sa présence proche et réelle (comme si la Mère de Dieu était restée parmi les hommes, qu'elle ne faisait que dormir ainsi que l'exprime l'hymne liturgique de la fête : «dans ta Dormition tu n'as pas abandonné le monde, ô Mère de Dieu »). Il y a toute une théologie derrière ce mot où la tradition orthodoxe souligne la proximité de la Mère de Dieu, et son intercession en faveur de l'homme auprès de Dieu.

\section{En guise de conclusion}

Ainsi, partant du modèle occidental, Dimitri de Rostov compose un tout autre texte où nous pouvons observer, par les choix de langage dramaturgique et théâtral qu'il fait, les nuances particulières de la perception orthodoxe de la fête de l'Assomption et du théâtre chrétien. Ce qui l'intéresse dans le traitement occidental du sujet de l'Assomption, ce n'est pas l'art d'enchaîner les épisodes en vue de renforcer la tension dramatique (les scènes de bousculade des juifs ou les pièges

\footnotetext{
1 M. Speranskij, préface, in Le Drame de la Dormition, op. cit

${ }^{2}$ J. C. Roberti. Orthodoxie et théâtre // Contacts, XXV année, № 83, 1973, p. 218
} 
ridicules des diables ${ }^{1}$ ), ce ne sont pas non plus les remarquables trouvailles scénographiques, mais les possibilités qu'offre le théâtre de présenter l'histoire sainte comme toujours actuelle, répondant aux questionnements de la modernité et éclairant le sens spirituel de la fête.

Pourtant, ces possibilités s'avèrent manifestement limitées. L'opposition ecclésiale au théâtre, pleinement connue en occident depuis Tertullien (II s.) jusqu'à Bossuet ${ }^{2}$, a pris dans la tradition orthodoxe russe une ampleur qui a empêché l'apparition même du théâtre spirituel. Cependant, l'exemple de Dimitri de Rostov montre que la conscience religieuse russe des $\mathrm{XV}^{\mathrm{e}}$ XVII ${ }^{\mathrm{e}}$ s. était ouverte aux spectacles chrétiens, comme en témoigne l'enthousiasme d'Abraham de Souzdal $^{3}$ devant les mystères joués à Florence. On peut certes trouver quelques auteurs parmi les intellectuels de cette époque (Siméon de Polotsk, L'Acte de la fournaise $(1673)^{4}$ ou les chefs de l'Eglise (Théophane Propokovich, tragédie Vladimir $(1705)^{5}$ mais ils sont restés des exceptions. L'idée d'un théâtre sacré qui essaie de garder l'« équilibre liturgique entre re/présentation et présentation "6 avec une approche intelligible correspond bien à la mentalité orthodoxe, mais se trouve en contradiction avec la nature même du théâtre et, par conséquent, n'est pas vraiment exploitée. Mais cette idée trouve sa pleine expression dans l'iconographie russe médiévale qui se développe simultanément à l'essor du théâtre des mystères en Occident.

Ainsi nos deux textes illustrent bien les deux voies divergentes que prend le genre du mystère en occident et en Russie : d'un côté, un développement brillant dont la tradition perdure aujourd'hui encore, de l'autre une existence modeste, qui se renforce dans les époques d'ouverture à l'occident, et qui semble d'ailleurs connaître un renouveau dans le cadre de la renaissance spirituelle dans la Russie contemporaine, où les mystères du métropolite de Rostov ont par exemple été mis en scène à Moscou (Rostovskoe dejstvo au Théâtre de la Pokrovka, 2005).

\footnotetext{
${ }^{1}$ absents dans le mystère d'Elx, mais présents dans la plupart des textes de ce type.

${ }^{2}$ Bossuet, Maximes et Réflexions sur le théâtre (1694).

${ }^{3}$ L'évêque Abraham de Souzdal faisait partie de la délégation russe au concile de Florence, en 1437-1440, et fut témoin des représentations des mystères de l'Annonciation et de l'Ascension de Feo Belcari. Il a laissé une description remarquable de ces représentations sacrées comme d'un «spectacle divinement monté», «habile et merveilleux, semblable à la descente des Cieux de l'Archange Gabriel vers Nazareth pour porter l'heureuse annonce à la Vierge Marie», in Hoždenie Avraamiâ Suzdal'skogo na Vos'moj Sobor s mitropolitom Isidorom // Kniga hoždenij, 1984, s. 333-336; Trudy otdela drevnerusskoj literatury, 33, 1979 - s. 28-36.

${ }^{4}$ Siméon de Polotsk (1629-1680), moine, poète et dramaturge du XVII siècle, est l'auteur du mystère Le Roi Nabuchodonosor, le veau d'or et les trois adolescents qui ne furent pas brûlés dans la fournaise (O Navuhodonosore tsare, o tele zlate i o triex otročex v peŝi ne sožžennyh)" (1673), dont la forme est celle du mystère occidental, qui était considéré comme un modèle par le théâtre russe naissant.

5 Théophane Prokopovitch (1681-1736), un des chefs de l'Eglise russe, a écrit une tragédie, Vladimir (1705), à propos de l'événement clé de l'histoire religieuse russe : le baptême de la Russie.

${ }^{6}$ J. C. Roberti. Orthodoxie et théâtre, op. cit, p. 220.
} 